\title{
El futbol y la seguridad social, caso \\ "Las tragedias de los estadios Hillsborough y Heysel"
}

Soccer and social security, "Hillsborough and Heysel stadiums tragedies" case

Pedro Melchor-Chávez ${ }^{a}$, Missael Melchor-Dorantes ${ }^{b}$, Ivette Flores-Jiménez ${ }^{c}$, Raúl RodríguezMoreno ${ }^{c}$, Miguel A. Vázquez-Alamilla ${ }^{c}$

\begin{abstract}
:
This document aims to answer the following research question. What is the importance of the Hillsborough and Heysel tragedies in the political and social process occurred in England in the twentieth century around football and social security? To understand this historical process, an analysis is made of the social and political causes of these tragedies, their development and the repercussions they had on society and the government. In addition, the actions taken to prevent similar tragedies in England. Primary and specialized sources are used, giving a historical, political and sports context. All this with the aim of analyzing and understanding how two tragic mass events can be the breaking point to change the culture and social security around a sport as popular as soccer, and how positive changes are achieved for short, medium and long term in a country. Concluding on how a government can make decisions that improve the environment that surround a country, both sports and social, if a cultural problem is attacked from root.
\end{abstract}

\section{Keywords:}

England, football, soccer, social security, tragedies

\section{Resumen:}

El presente documento pretende dar respuesta a la pregunta de investigación, ¿Cuál es la importancia de las tragedias de Hillsborough y Heysel dentro del proceso político y social ocurrido en el siglo XX en Inglaterra alrededor del futbol y la seguridad social? Para comprender dicho proceso histórico, se realiza un análisis de las causas sociales y políticas de dichas tragedias, el desarrollo de éstas y las repercusiones que tuvieron en la sociedad y el gobierno, además de las medidas que se tomaron para evitar que se repitieran tragedias similares en Inglaterra. Se usan fuentes primarias y especializadas en el tema, dando un contexto histórico, político y deportivo. Todo esto con el objetivo de analizar y comprender cómo dos eventos luctuosos masivos pueden ser el punto de quiebre para cambiar la cultura y la seguridad social que rodean a un deporte tan popular como el futbol, y cómo se logran cambios positivos para corto, mediano y largo plazo en una nación. Para después concluir en cómo un gobierno puede tomar decisiones que mejoran el ambiente que se vive en un país, tanto en lo deportivo y en lo social, si se ataca un problema cultural de raíz.

\section{Palabras Clave:}

Estadio, futbol, Inglaterra, seguridad social, tragedia

\section{Introducción}

Cuando se habla de la historia del deporte, y específicamente del futbol, lo primero en lo que se piensa son las hazañas y las victorias que

\footnotetext{
Autor de Correspondencia, Universidad Autónoma del Estado de Hidalgo, Escuela Superior de Tlahuelilpan, Email: pedro_melchor6320@uaeh.edu.mx

b Alumno del Instituto Tecnológico Autónomo de México, Dirección Financiera. Email: mmd.leassim@gmail.com

c Universidad Autónoma del Estado de Hidalgo, Escuela Superior de Tlahuelilpan, Ivette Flores Jiménez: ivette_flores7 @ hotmail.com; Raúl Rodríguez Moreno: raromo@hotmail.com; Miguel Ángel Vázquez Alamilla: m_vazquez_alamilla@hotmail.com
} 
determinados equipos han realizado a lo largo del tiempo, se trata de aficiones incondicionales y de jugadores que se vuelven memorables por sus actuaciones. Pero en la historia del futbol también hay tragedias, las cuales no sólo quedan en la memoria de los aficionados al futbol, sino en la de toda una sociedad que queda marcada por las muertes que ocurren en éstas. Toda tragedia 0 evento histórico relacionado con muertes humanas tiene un proceso con causas, antecedentes y consecuencias. Dentro de esta investigación se pretende dar respuesta a la siguiente pregunta de investigación ¿Cuál es la importancia de las tragedias de Hillsborough y Heysel dentro del proceso político y social ocurrido en el siglo XX, en Inglaterra alrededor del futbol y la seguridad social? Se analizan las causas y antecedentes que tuvieron ambas tragedias desde las políticas y las sociales. Se hace una recopilación de información de lo ocurrido en ellas a través de distintas fuentes especializadas, y se verá qué consecuencias existieron a partir de las mismas tragedias. Para así, poder concluir qué y por qué ocurrió en este proceso histórico inglés y entender la importancia de ambas tragedias en dicho proceso. Además de poder puntualizar la relevancia de las decisiones tomadas por el gobierno para afrontar la inseguridad social vivida en Inglaterra alrededor del futbol. El presente estudio surge a partir de la inquietud por relacionar un deporte que mueve masas, como el futbol con la historia de una nación, por visualizar la relevancia que algunas tragedias ocurridas en un ambiente deportivo pueden alcanzar a nivel político y social, e incluso cómo pueden llegar a trascender como puntos coyunturales un proceso histórico importante a nivel continental, e inclusive global.

\section{Desarrollo}

Durante la primera mitad del siglo XX Inglaterra se vio afectada por el desempleo, debido a las consecuencias negativas, económicas y sociales, que dejó el periodo de guerras. El $12 \%$ de la población fue afectado por la falta de oportunidades. La pobreza aumentó de manera significativa gracias a esto. La educación era escaza dentro de las clases media y baja, pues los jóvenes desde los doce años tenían que trabajar para sustentar los gastos de la casa. Estos niños y jóvenes crecieron con la opresión del sistema capitalista, obligados a hacer jornadas laborales largas y recibiendo un salario bajo, estaban ansiosos por manifestar su inconformidad con la autoridad. Hacia la segunda mitad del siglo XX, la clase trabajadora seguía viviendo en condiciones deplorables y en la falta de oportunidades. El gobierno inglés tomó medidas de austeridad ante la crisis económica que se estaba viviendo entre los años 1970 y 1980, acción que alteró más a la parte pobre de la sociedad, así, con el pretexto de apoyar a un equipo de futbol los jóvenes comenzaron a manifestarse violentamente dentro y fuera de los estadios para expresar que su modus vivendi era la violencia, ya que el trabajo leal y la tranquilidad no les había brindado nada, así nacieron los Hooligans. 10,11,16,20

Ante la amenaza de los Hooligans, los equipos europeos decidieron levantar rejas para separar el terreno de juego de la afición, quitaron las butacas de algunas partes de los estadios para evitar que fueran usadas como proyectiles, pidieron a la policía que fueran más exhaustivos buscando posibles armas escondidas en los cuerpos de los Hooligans y que fueran más severos al tratar con personas violentadas. Pero más que soluciones a la inseguridad que se estaba viviendo en los estadios, estas decisiones administrativas dieron pie a más incertidumbre y problemas. El futbol en Inglaterra empezaba a ser considerado un evento lleno de riesgos para los espectadores. 20

Tragedia de Heysel (1985)

El 29 de mayo de 1985 se llevó a cabo la final de la UEFA Cup, el torneo más importante para los clubes europeos de la época. La final tomó lugar en el Heysel Stadium ubicado en Bruselas, Bélgica. Los participantes en este partido serían la Juventus de Turín, Italia y el Liverpool F.C. de Inglaterra. Las expectativas eran altas por el partido y por la cantidad de gente que había viajado desde Italia e Inglaterra para presenciar el partido. Entre estos espectadores se encontraban dos de las porras más violentas de Europa, los 
Ultras italianos y los Hooligans ingleses. Desde horas anteriores al partido la ciudad de Bruselas se había llenado de altercados provocados por Hooligans en estado de ebriedad, las mujeres eran acosadas sexualmente, ocurrieron robos a autos y transeúntes, bares y restaurantes fueron destrozados, y se asaltaron joyerías, entre otros comercios. "Los hinchas británicos dejaron el centro de Bruselas como si una horda de salvajes hubiera pasado por alli". 1,13,14

Ya dentro del estadio, 45 minutos antes de que empezara el partido, el mayor conflicto se desató. Un grupo de Hooligans que se encontraba en la zona $X$ del estadio se lanzó al ataque contra un sector de Ultras que se encontraban en la zona $Z$ (Ver Figura 1.), haciendo uso de proyectiles, botellas y navajas. Estos dos grupos se encontraban separados por una línea de policías, pero en una cantidad no suficiente para detener los embates de los Hooligans, que acorralaron a los Ultras entre un muro de protección del estadio y las rejas que daban al campo de juego. La avalancha de Hooligans fue tan grande que el muro de contención de la zona $Z$, que era bastante viejo, se derrumbó, haciendo la estampida humana mayor. Esto dejó personas enterradas bajo los cuerpos de otros, provocando la asfixia y sofocación de los que corrieron la suerte de estar por debajo. 1,4

Al derrumbarse el muro de la zona $Z$ dio pie a que algunos aficionados de la Juventus escaparan de la avalancha y pudieran ayudar a otros a salir del problema. Los cuerpos de seguridad y de emergencia fueron insuficientes para evitar la tragedia, 39 personas murieron en el incidente, la mayoría de ellos italianos, además hubo más de 350 lesionados. El partido se jugó de todas formas, a pesar del malestar de los jugadores al saltar al campo de juego y ver un grupo de gente lesionada y muerta sobre el terreno de juego. El resultado de los incidentes ocurridos en el Heysel Stadium fue una sanción para todos los clubes ingleses vetados de territorio belga, además de estar fuera de competiciones europeas por cinco años; el Liverpool F.C fue sancionado por un año más. Pero estas sanciones no terminaron con los problemas en los estadios de equipos ingleses, ni la inseguridad social que rodeaba al futbol. 3,5,14

Tragedia de Hillsborough (1989)

Cuatro años después de la tragedia de Heysel, ocurriría otro incidente que marcó de luto la historia del futbol inglés. El 15 de abril de 1989 se jugaría la semifinal de la FA Cup. Los equipos involucrados fueron el Nottingham Forrest F.C. y el Liverpool F.C. Como era un partido de eliminación directa, se tendría que jugar en un campo neutral, el escogido por la FA (Football Association) fue el Hillsborough Stadium ubicado en Sheffield. Las aficiones de los equipos fueron llegando desde el mediodía a la localidad. 9

El problema comenzó cuando se dio a conocer la forma de dividir aficiones por parte de la policía, los seguidores del Nottingham Forrest fueron colocados en la parte sur y este del estadio, las cuales eran las más amplias de éste. Para evitar cualquier conflicto entre aficiones y Hooligans, la porra del Liverpool fue ubicada en las zonas oeste y norte del estadio, a las cuales sólo se tenía acceso a través de la avenida Leppings Lane (Ver Figura 2.) y los torniquetes que daban a las puertas $A, B$ y $C$, la última, que era la más amplia de las tres, se encontraba cerrada, pues su uso era exclusivamente para la salida. Conforme se acercaba la hora del partido un cúmulo de gente rodeaba Leppings Lane, puesto que el acceso al estadio era lento. Las terrazas 3 y 4 comenzaron a llenarse, se estimaba tenían capacidad para 2200 personas, pero en investigaciones posteriores se vería que una remodelación redujo ésta a sólo 1600 personas. 5,17

A las 2:52 horas, los equipos saltaron a la cancha y los gritos de la gente que ya estaba adentro del estadio generaron histeria en los que aún se encontraban afuera. Para aliviar los empujones y amontonamientos, la policía dio la orden de abrir la puerta $C$, dejando entrar a más de 2000 aficionados que, a través del túnel mayor, se dirigirían a las terrazas 3 y 4 . El flujo era accidentado, pero seguía dando entrada a la gente, hasta que 3000 aficionados ya se encontraban en las terrazas centrales. La gente 
que había entrado en horas tempranas estaba siendo aplastada en contra de la reja que daba al campo de juego y contra las rejas laterales. Mientras el partido daba inicio, un muro de contención de la terraza 3 se desplomó, generando una avalancha de cuerpos, los aficionados comenzaron a brincar hacia el campo de juego y a las terrazas contiguas para ponerse a salvo. A los seis minutos de juego el partido se detuvo por el caos ocasionado. Los cuerpos de emergencia fueron llegando al estadio tratando de rescatar a los que se asfixiaban y conmocionaban con el peso de la gente, y tratando de sanar a los lesionados. Pero el cuerpo de policía sólo permitió el acceso a una de las ambulancias de las que llegaron al estadio, haciendo aún más difícil el rescate de heridos. 5,9

El partido fue calendarizado para otro día, aunque los resultados finales del evento fueron 95 muertos (una persona más moriría cuatro años después por la misma conmoción sufrida ese día) y más de 700 heridos. Además que esta tragedia dio pie a una investigación a fondo de lo que pasó y lo que provocó el accidente, el Informe Taylor, éste se tuvo que realizar puesto que los primeros reportes de la policía culpaban a gente en estado de ebriedad y a Hooligans, pero omitían la negligencia policial y la falta de organización que realmente causaron el incidente. También se dio paso a un análisis de lo que se debería de mejorar en la seguridad social dentro de los eventos deportivos en Inglaterra, el Football Spectator Act. 5,9

\section{Football Spectator Act y Taylor Report}

Después de ambas tragedias, sumadas a otra que ocurrió en Valley Parade en 1985 donde Hooligans incendiaron las gradas de madera, el gobierno inglés que era liderado por Margaret Tatcher lanzó dos documentos que cambiarían el ambiente en el que se vivían los partidos de futbol y eventos deportivos.

El primero fue el Football Spectator Act, una legislación del Parlamento que, aunque estaba siendo propuesta meses antes de que ocurriera la tragedia de Hillsborough, no vio la luz hasta después de ésta. El documento se divide en tres partes dónde se marcan las acciones permitidas y no permitidas dentro y fuera de los estadios en el territorio inglés y galés. También se explican las sanciones que entrarán en vigor en caso de que algún ciudadano o administrativo de un equipo se vea involucrado en problemas de violencia e inseguridad relacionadas al deporte. Además de aclarar que habría préstamos a los clubes por parte del gobierno para solventar gastos necesarios para los cambios. Entre los puntos más destacados y controversiales de la legislación se encuentran estos dos: 2,12

- "Sección 14 A: Cualquier persona que cometa una ofensa hacia el deporte y los aficionados a éste y sea demostrada su culpabilidad, será vetada de cualquier evento deportivo que se lleve a cabo en territorio inglés y galés"

- "Sección 14 B: Las autoridades correspondientes a los eventos deportivos quedan autorizadas a vetar y alejar del estadio a cualquier persona que sea identificada con perfil violento y amenazante a la seguridad de terceros, sin necesidad de que se le encuentre culpable de alguna ofensa al deporte". 2

Ambos puntos fueron claves en la prevención de accidentes y conflictos causados por Hooligans. Pero, como se puede observar, afectaban derechos humanos como la recreación, al no dar una segunda oportunidad a los que hayan participado en ofensas, y a la libertad de expresión, ya que la autoridad podía juzgar a través de prejuicios y no acciones cometidas por los aficionados. Ante esta controversia en el año 2000 con el Football (Disorder) Act se reformó la sección $14 \mathrm{~A}$ dando un máximo de 3 años al veto, y de 6 a 10 años en caso de haber sido un delito grave. Al mismo tiempo se dio un máximo de 2 a 3 años al veto aplicado a las personas castigadas por la sección 14 B. 2

El segundo documento y más importante, fue The Hillsborough Stadium Disaster, mejor conocido como el Taylor Report, siendo un reporte escrito por Lord Justice Taylor publicado finalmente en 1990 y dividido en dos partes. La primera es una 
investigación a fondo sobre lo ocurrido en el Hillsborough Stadium, dónde se le quitan responsabilidades a la afición por la tragedia y se inculpa al cuerpo policiaco de Yorkshire por sus errores y negligencia. La segunda parte es una compilación de recomendaciones hechas por Taylor hacia los aficionados, el gobierno y las administraciones de los equipos de futbol profesional inglés para mejorar el ambiente que rodeaba al futbol. Entre las recomendaciones destacadas y puestas en acción fueron: 6,17

- Prohibir la existencia de estadios con localidades a pie, esto con el fin de prevenir avalanchas humanas por desbordes. Colocación de asientos individuales en la totalidad del recinto, salvo en palcos preferentes y cabinas privadas cerradas. Asimismo, se prohíbe la existencia de rejas alrededor del campo de juego, esto con el fin de permitir tener una vía de escape hacia la cancha en caso de tragedia.

- Prohibir el ingreso de asistentes bajo los efectos del alcohol o alguna otra droga. Asimismo, se recomienda no vender bebidas alcohólicas ni en los recintos, ni en un radio menor a diez calles.

- Colocación de cámaras permanentes y operativas en todo el recinto, con la suficiente calidad de imagen como para ser usadas como material de prueba en caso de juicio. 7

- Uso de guardias privados con entrenamiento en manejo de masas, primeros auxilios y seguridad. Reemplazando así a la policía dentro del recinto. Con motivo de destinar fuerza policial a las calles cercanas al estadio y en todo el recorrido de los asistentes.

- Prohibir la venta de boletos de entrada el mismo día del partido, con el fin de evitar aglomeraciones que pudiesen redundar en incidentes mayores.

- Libertad al derecho de admisión hacia los clubes, para permitir o no el ingreso de los fanáticos, tanto locales como visitantes al estadio. Con ello, permite la existencia de un registro base de aficionados con el fin de mantenerlos ubicables.

- El precio del boletaje debe aumentarse y un mayor porcentaje de boletos deben ser vendidos como abonos por temporada completa, evitando que cualquier persona entre a hacer desfiguros al estadio. 20

- Crear una organización que monitoreé constantemente el estado de las instalaciones deportivas y las licencias de los sistemas de seguridad en los estadios. (SGSA: Sports Grounds Safety Authority, antes FLA: Football Licensing Autorithy). 15

Con estas recomendaciones llevándose a cabo comenzaron a haber resultados positivos dentro y fuera de los estadios y clubes de futbol.

\section{Consecuencias y actualidad}

A partir de los ya mencionados, Reporte Taylor y el Football Spectator Act, comenzaron a darse cambios en el ambiente que rodeaba los partidos de futbol y existieron procesos que llegarían a dar confort y seguridad a los aficionados en territorio inglés y galés. Ahora, a partir de los puntos anteriormente analizados y los próximos a analizar podemos empezar a ver ¿Importancia de las tragedias de Hillsborough y Heysel dentro del proceso político y social ocurrido en el siglo $\mathrm{XX}$, en Inglaterra alrededor del futbol y la seguridad social?

Al subir los precios de los boletos casi al triple, los clubes provocaron que la clase trabajadora tuviera menos acceso a los partidos, como consecuencia la asistencia de Hooligans disminuyó notablemente, la mayoría de éstos optaron por ver los partidos desde bares o en sus hogares. $Y$ en caso de que existiera la asistencia de los Hooligans la seguridad ya era más eficaz y los castigos a los que hicieran desfiguros, mayores. A partir de los ingresos que recibieron los equipos con los boletos, los préstamos gubernamentales, la creación oficial de la división Premier de Inglaterra, y el contrato millonario que la empresa Sky ofreció por la transmisión de partidos, existió un Boom económico para el año 1992. Esto permitió mayor inversión a los inmuebles, a la seguridad y terminar de pagar las deudas generadas al inicio. La UEFA (Union of European Football Associations) comenzó a imitar las medidas tomadas por Inglaterra y hacerlas 
obligatorias de igual manera en el resto de Europa. La FLA (Football licensing Autorithy), que años después se convertiría en la SGSA, se encargó de monitorear los setenta y cuatro estadios más importantes del territorio para que toda la tribuna tuviera asientos, se encargó de revisar los circuitos cerrados de video de los estadios y se dio a la tarea de ir persuadiendo la cultura de la seguridad en los aficionados de cada ciudad, se encargó de entrenar y actualizar a los cuerpos privados de seguridad que se implantaban en cada inmueble y constató que las rejas de seguridad fueran removidas de cada estadio de futbol profesional. 6,7,15,20

Hay que remarcar que las mejoras en seguridad fueron evolucionando de acuerdo a las necesidades del público por lo que se publicaron más documentos relacionados al futbol como: Public Order Act 1986; Football Spectators Act 1989; Football Offences Act 1991; Criminal Justice and Public Order Act 1994; Football Disorder Act 2000; Violent Crime Reduction Act 2006. Así, año tras año la seguridad en los partidos iría mejorando. 8 ,

Para la temporada 2003-2004 del futbol inglés, The UK Home Office (Interior Minister) publicó las estadísticas sobre la violencia en partidos de futbol, entre los datos más interesantes están: 2

- Los arrestos relacionados con ofensas al fútbol se han reducido en un $10 \%$ comparado con el 2003, de 4413 a 3982.

- Los niveles de arresto permanecen bajos. Esta temporada se vio la mayor asistencia por parte del público 29'197,510 personas asistieron y se hicieron efectivos sólo 3,010 arrestos, siendo esto el $0.01 \%$ de aficionados.

- El $57 \%$ de los arrestos se hicieron fuera del estadio, $87 \%$ de los arrestos fueron por desorden violento afuera del estadio.

- Los vetos a personas violentas han sido más efectivos y en mayor cantidad, gracias al accionar preciso de la policía identificando sujetos. En agosto de 2003 existían 1794 vetos, para octubre de 2004 la cifra subió a 2596. 2
Para 2013 la imagen de los aficionados ingleses y la seguridad social que rodea los estadios era diferente a la de la década de 1980, los estadios lucen tranquilos y ordenados, los arrestos fuera y dentro de inmuebles deportivos son bajos, la imagen de Inglaterra a nivel internacional ya no es relacionada con violencia, y la Premier League es la liga más lucrativa a nivel mundial con ganancias registradas en 3.1 billones de libras (4.9 billones de dólares). Aunque no todo ha sido perfección. De igual forma, han existido críticas al modelo de seguridad, ya que el aficionado promedio batalla bastante para conseguir el dinero de las entradas y al ser las clases bajas y medias-bajas las más apasionadas a sus equipos los estadios no son tan cálidos para el equipo local como solían serlo años atrás. El dilema ahora está entre la pasión, la afición y la seguridad. 8,20

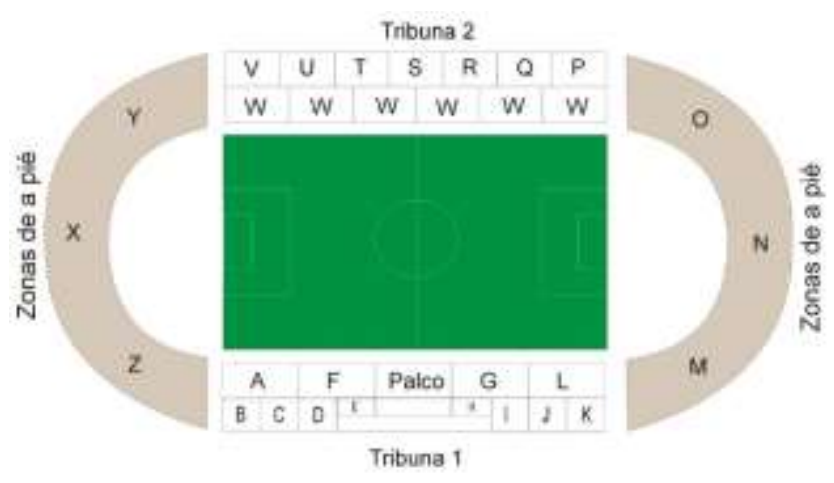

Figura 1. Esquema del Heysel Stadium para la final Juventus vs Liverpool 1985. 18

\section{Conclusiones}

Para responder la pregunta de investigación ¿Cuál es la importancia de las tragedias de Hillsborough y Heysel dentro del proceso político y social ocurrido en el siglo $X X$, en Inglaterra alrededor del futbol y la seguridad social? Vemos que, como ya ha sido expuesto en esta investigación, dentro de la Inglaterra del siglo $X X$ se vivió un proceso social y político alrededor del futbol, que resultó en una cultura de seguridad y positivismo por parte de los asistentes a los partidos. El presente del deporte en Inglaterra demuestra la tranquilidad y seguridad que resultó de un proceso que incluyó problemas sociales, 
violencia, muertes, malestar generalizado $\mathrm{y}$ decisiones políticas.

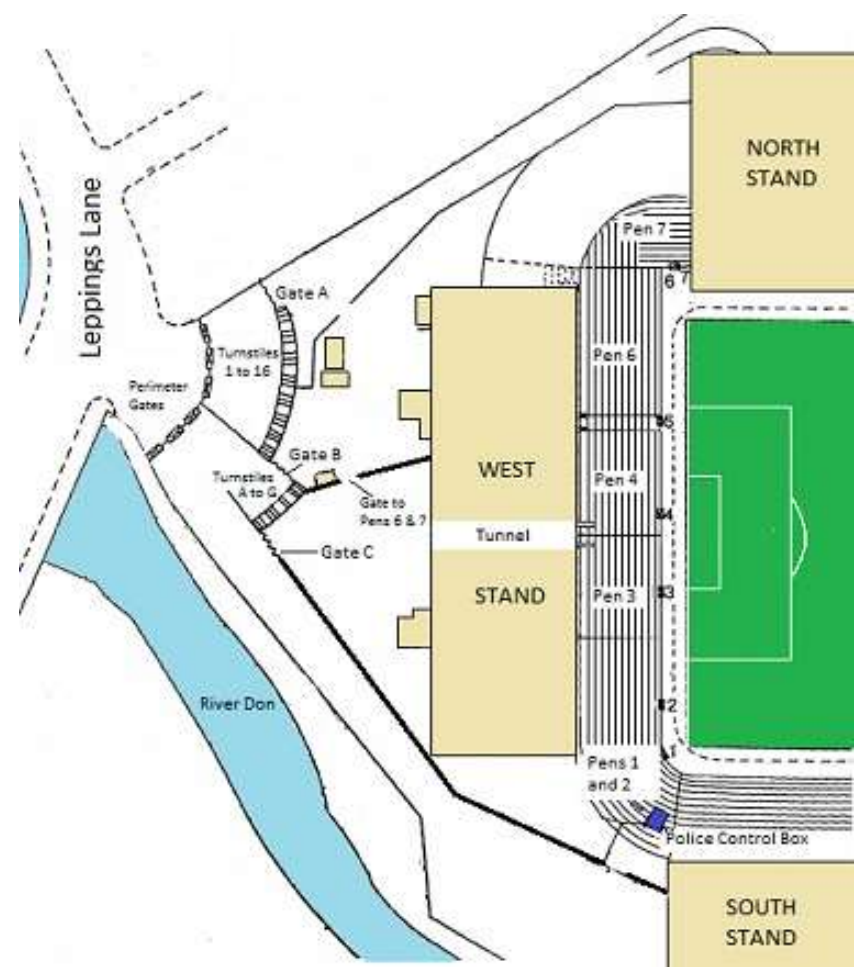

Figura 2. Esquema del Hillsborough Stadium. 19

La situación de la clase media y baja de la población en la primera mitad del siglo XX provocada por los cambios sociales y decisiones políticas ante las crisis económicas, fue el factor que alteró la estabilidad en la que se debe jugar y observar el deporte. Nació la ideología Hooligan y se esparció rápidamente en el territorio inglés, provocando que el mismo gobierno y los clubes tomaran medidas tan precipitadas que con el paso del tiempo se volvieron contraproducentes, entre ellas levantar rejas para dividir el campo de juego de la afición, darle demasiado poder a la policía, y quitar los asientos en ciertas partes de la tribuna. Dos tragedias que cobraron casi 200 vidas y dejaron cientos más de lesionados, terminaron siendo el espejo de lo que se hizo mal en el gobierno, en los clubes y en la afición. Tuvieron que llegar cambios atacando la raíz del problema, y aunque tuvieron tintes de clasistas, dieron resultados al brindar tranquilidad a la gente que asistía con miedo a los estadios.
A partir de las tragedias, Inglaterra se ha convertido en el ejemplo a seguir para los países que sufren de violencia en los estadios. El gobierno y los clubes aceptaron y analizaron los errores cometidos, los cuales quedarán en la memoria luctuosa del país y comenzaron un proceso normativo y de limpieza que dio pie a un cambio de imagen a nivel internacional. Las tragedias fueron coyunturales para que el cambio existiera. Pero no bastó con apaciguar el problema de seguridad social a corto plazo con el Taylor Report y el Football Spectator Act, sino que se determinó a dar mejores resultados a mediano plazo, con las legislaciones posteriores (Public Order Act 1986; Football Spectators Act 1989; Football Offences Act 1991; Criminal Justice and Public Order Act 1994; Football Disorder Act 2000; Violent Crime Reduction Act 2006), y a dejar un legado firme para que a largo plazo, con la SGSA, se creara una cultura sana dentro y fuera de los estadios y se erradicara completamente la violencia que rodeaba al deporte más arraigado a la cultura popular inglesa, el futbol.

Si bien, la presente investigación se vale de un número amplio de fuentes primarias $y$ especializadas, se pueden encontrar algunas limitaciones tanto en la lejanía geográfica y en el tiempo que hay entre los sucesos y la realización de este proyecto. De igual forma, la traducción de las fuentes que están originalmente en inglés puede cambiar el contexto de algunas oraciones y se puede interpretar de diferente manera lo que los entrevistados en los documentales y los autores de las fuentes primarias refieren. Se debe de tomar en cuenta la naturaleza histórica de este trabajo y valerse de saber que los autores de las fuentes especializadas también basan sus textos en interpretaciones y posturas ideológicas. Pero la visión global de este texto, se basa en la comparación y contrastación de fuentes, que, aunque pueden no ser provenientes de todos los puntos de vista relacionados al tema, dan una idea global de la relevancia de las tragedias en la historia inglesa. 


\section{Referencias}

[1] ABC, Diario. (s.f.). Heysel, la masácre que cambió el futbol. (D. ABC, Ed.) ABC, Diario. Obtenido de http://www.abc.es/20100610/archivo-historia-abc/heyselcastastrofe-bruselas-liverpool-201006101230.html.

[2] Asser Institute. (s.f.). www.asser.nl. Obtenido de http://www.asser.nl:

http://www.asser.nl/default.aspx?site_id=11\&level1=13910\&1 evel $2=13974 \&$ level $3=\&$ textid $=36384$

[3] BBC. (1985). English teams banned after Heysel. Obtenido de http://news.bbc.co.uk/onthisday/hi/dates/stories/may/31/newsi d_2481000/2481723.stm

[4] BBC. (s.f.). Heysel 1985: Requiem for a Cup Final.

[5] BCC. (s.f.). How The Hillsborough Disaster Happened. Obtenido de http://news.bbc.co.uk/2/hi/uk_news/7992845.stm

[6] Betancourt, A. (s.f.). El Informe Taylor: hacía un fútbol sin violencia. Fútbol y violencias, 126-127.

[7] charlatecnica.cl. (2012). charlatecnica.cl. Obtenido de http://charlatecnica.cl/2012/02/football-spectators-act-el-antesy-el-despues-en-la-premier-league/

[8] Giulianotti, R. (2013). Social and legal factors in the decline of English football hooliganism. International Center for Sport Security, 1(3), 33-37. Obtenido de https://issuu.com/the_icss/docs/icss-journal-1-3

[9] Gordon, D. (Dirección). (2014). Hillsborough [Película].

[10] Humphries, S. (1995). Hooligans or Rebels? Oral History of Working Class Childhood and Youth, 1889-1939.

[11] Lambert, T. (2014). Daily life in Britain in the 20th century. Obtenido de http://www.localhistories.org/20thcent.html

[12] legislation.gov.uk. (1989). http://www.legislation.gov.uk/ukpga/1989/37/contents. Obtenido de Football Spectators Act 1989: http://www.legislation.gov.uk/ukpga/1989/37/contents

[13] libcom.org. (s.f.). History of the Ultra movement in Italy. Obtenido de libcom.org: https://libcom.org/library/historyultra-movement-italy

[14] Mosqueda, J., Salinas, Á., \& López, Á. (2006). Tragedia en Heysel. Futbol Total: 50 escándalos del balompié, 34-35.

[15] SGSA. (s.f.). Sports Grounds Safety Authority. Obtenido de http://www.safetyatsportsgrounds.org.uk

[16] Shaw, M., \& McCulloch, K. (2009). Hooligans or rebels? Thinking more critically about citizenship. Youth \& Policy, 101, 5-9.

[17] Taylor, P. (1990). The Hillsborough Stadium Disaster (Taylor Report). Peter Taylor, Baron Taylor of Gosforth.
[18] wikipedia.org. (s.f.). es.wikipedia.org. Obtenido de http://es.wikipedia.org/wiki/Tragedia_de_Heysel\#mediaviewer /Archivo:Heysel.jpg

[19] wikipedia.org. (s.f.). es.wikipedia.org. Obtenido de http://en.wikipedia.org/wiki/Hillsborough_disaster\#mediaview er/File:Hillsborough_west_side_1989.png

[20] www.futboldecafe.com. (s.f.). www.futboldecafe.com. Obtenido de http://www.futboldecafe.com/miscelaneo/estadios/informetaylor-y-el-manejo-de-multitudes-sacrificar-aficion-poratrapar-radicales/ 